\title{
Varicella vaccine without human serum albumin versus licensed varicella vaccine in children during the second year of life: a randomized, double-blind, non-inferiority trial
}

Roman Prymula ${ }^{1 *}$, Robert Simko ${ }^{2}$, Michael Povey $^{3}$ and Andrea Kulcsar ${ }^{4}$

\begin{abstract}
Background: GSK's varicella vaccine contains human serum albumin (HSA) which is used to stabilize the virus and prevent immunogens from adhering to the injection vial walls. However, because HSA is derived from human blood, there is a theoretical risk that it might contain infectious agents which could be unsafe for humans. Given this concern, a study was undertaken to compare the immunogenicity and safety of a new formulation without HSA with the currently licensed varicella vaccine in the Czech Republic and Hungary.

Methods: Healthy children aged 11-21 months received two doses of the varicella vaccine either with or without HSA. Antibody titres against varicella-zoster virus (anti-VZV) were measured 42 days after each dose, using an immunofluorescence assay (IFA, cut-off $=4$ dilution $^{-1}$ ) and enzyme linked immunosorbent assay (ELISA, cut-off $=25 \mathrm{mlU} / \mathrm{ml}$ ). Solicited local symptoms were recorded during a 4-day post-vaccination follow-up period; solicited general and unsolicited symptoms were recorded during a 43-day post-vaccination follow-up period and serious adverse event (SAEs) were recorded throughout the study.

Results: Of 244 children (mean age $=15.2$ months [SD = 3.2]) vaccinated in the study, 233 (vaccine without HSA $N=117$; vaccine containing HSA N =116) formed the according-to-protocol immunogenicity cohort. Observed seroconversion/seroresponse rates were $>98$ and $100 \%, 42$ days after doses 1 and 2, respectively. The rates were within the same range in both groups, irrespective of the testing assay. The varicella vaccine without HSA was non-inferior to the licensed vaccine in terms of anti-VZV antibody Geometric Mean Titre/ Concentration ratio (1.12 [95 \% Cl:0.86-1.46] by IFA; 1.12 [95 \% Cl:0.93-1.33] by ELISA) approximately six weeks after the first dose of the 2-dose vaccination course. The incidence of solicited and unsolicited symptoms was similar after both vaccines; low-grade fever was numerically higher after the first dose of the varicella vaccine without HSA. Seven SAEs were reported, none of which were fatal or considered to be vaccine-related.
\end{abstract}

Conclusions: The first dose of a new varicella vaccine without HSA was immunologically non-inferior to the licensed varicella vaccine. After two doses, both vaccines had acceptable safety profiles in children aged 11-21 months in the Czech Republic and Hungary.

Trial registration: NCT00568334, registered on 5 December 2007 (www.clinicaltrials.gov).

Keywords: Varicella vaccine, Non-inferiority, Human serum albumin, HSA

\footnotetext{
* Correspondence: prymula@seznam.cz

${ }^{1}$ Faculty of Military Health Sciences, University of Defence, Trebesska 1575,

50001 Hradec Kralove, Czech Republic

Full list of author information is available at the end of the article
} 


\section{Background}

In children, primary infection with varicella-zoster virus (VZV) causes chicken pox [1], a highly contagious but mostly benign disease. It can, however, be associated with a range of serious complications such as secondary bacterial infections, pneumonia, cerebellar ataxia, encephalitis and Reye's syndrome [1]. In adolescents and adults, primary VZV infection causes greater morbidity and mortality [2]. Following primary VZV infection, the latent virus persists in the central nervous system and can be reactivated in later life to cause herpes zoster (HZ) or shingles [2, 3]. Complications of HZ include herpes ophthalmicus, cutaneous dissemination, pneumonia and other central nervous system, pulmonary, hepatic and pancreatic complications [4].

In Europe, over 90 \% of children contract chicken pox in the first 12 years of life [5-9]. Although the reported incidence of VZV-related deaths $(\leq 0.05 / 100,000$ population/ year) and hospitalizations (1.1-18.7/100,000 population/ year) in European countries is low, the burden of disease is nevertheless significant [10-12].

Two monovalent varicella vaccines are currently available in Europe: Varilrix ${ }^{\mathrm{Tm}}$ (GSK Vaccines) and Varivax ${ }^{\text {тм }}$ (Merck \& Co. Inc), both of which are derived from the Japanese Oka strain. Placebo-controlled clinical trials have demonstrated that both vaccines are immunogenic, safe and efficacious [13, 14]. A recent observer-blind study in Europe showed $65.4 \%$ efficacy for one varicella vaccine dose at preventing confirmed varicella of any severity, and $90.7 \%$ efficacy at preventing moderate to severe cases during 3 years of follow-up [15].

Since the World Health Organization recommendation for routine vaccination against childhood VZV infection in 1998 [16], several countries have implemented either of the varicella vaccines into their national immunization programs [17-24]. By 2008, the human serum albumin (HSA)-containing varicella vaccine $\left(\right.$ Varilrix $\left.^{\mathrm{TM}}\right)$ was licensed in 92 countries [25].

HSA is incorporated in live, attenuated vaccines to stabilize the virus and prevent immunogens from adhering to the walls of the injection vial. However, because HSA is a protein derived from human blood, there is a theoretical risk that it might contain infectious agents [26] which could make it unsafe for humans. Given this concern, the manufacturing process has been modified to remove HSA from the vaccine stabilizer added towards the end of production. This is in line with the European Medicines Agency recommendation to eliminate all blood-derived products of human origin from vaccine manufacturing [27]. This study was therefore undertaken to confirm that the varicella vaccine without HSA was immunologically non-inferior to the licensed varicella vaccine. The safety profile of both vaccines was also assessed despite the process change.

\section{Methods}

\section{Study design and subjects}

This phase II, non-inferiority study was conducted at 14 centres (offices of primary care physicians and general practitioners) in the Czech Republic (2 centres) and Hungary (12 centres) between November 2007 and April 2008 (NCT00568334). The primary care physicians recruited children and depending on the centre, either the physician/nurse administered the vaccine. The study was carried out in a double-blind manner, meaning that the investigators, study staff and parents/guardians of children were unaware of the vaccine given.

Healthy children 11-21 months of age were enrolled and randomized (1:1) to receive either two doses of varicella vaccine without HSA (Group A) or two doses of varicella vaccine containing HSA, Varilrix ${ }^{\mathrm{TM}}$ (Group B) which is licensed for the prevention of varicella in healthy children from 9 months of age. The selection criteria in this study were similar to previous studies conducted with the licensed vaccine $[28,29]$. The two doses were administered 42-56 days apart. Children were excluded from the study if they had received any live attenuated viral routine vaccines or investigational drug/vaccine within 30 days or immunosuppressant/immune modifying drugs/blood-derived products within six months of receiving the study vaccine. They were also excluded if they had been previously exposed to VZV or had: any confirmed or suspected immunosuppressive condition; a family history of immunodeficiency; chronic illnesses, allergies to any vaccine components or an acute disease at the time of enrolment. In order to account for the multiple routine vaccinations recommended at this age, children were allowed to receive routine inactivated vaccines up to eight days before each study vaccination so as to not interfere with the reactogenicity assessment after varicella vaccination. It was advised that children participating in the study should avoid the use of aspirin for six weeks after vaccination as Reye's syndrome has been reported following the use of aspirin during natural varicella infection.

All study-related documents including the study protocol were reviewed and approved by the Pardubice Regional Hospital and the Regional Hospital Nachod for the two participating centres, respectively, in the Czech Republic and by the Egészségügyi Tudományos Tanács Klinikai Farmakológiai Etikai Bizottság for the 14 participating centres in Hungary. The study was conducted in accordance with Good Clinical Practice, the Declaration of Helsinki and all applicable regulatory requirements. Parents/guardians provided written informed consent before any study procedures were performed.

\section{Study vaccines}

Both varicella vaccines were developed and manufactured by GSK Vaccines, Belgium. The potency of the 
$\mathrm{Oka} / \mathrm{RIT}$ strain in the licensed varicella vaccine was $10^{4.4}$ plaque forming units (pfu) $/ 0.5 \mathrm{~mL}$ compared with $10^{4.3}$ $\mathrm{pfu} / 0.5 \mathrm{~mL}$ in the formulation without HSA. Both vaccines were presented as lyophilized pellets, which were reconstituted with the supplied diluent before subcutaneous injection in the left upper arm.

\section{Immunogenicity}

Blood samples were collected immediately before vaccination and 42 days after each vaccine dose. Antibody titres against VZV (anti-VZV) were measured using two assays: a commercial enzyme linked immunosorbent assay (ELISA kit, Enzygnost ${ }^{\mathrm{TM}}$, Dade Behring, Marburg, Germany; assay cut-off $=25 \mathrm{mIU} / \mathrm{ml}$ ) and an immunofluorescence assay (IFA, Virgo ${ }^{\mathrm{TM}}$, Hemagen Diagnostics, Columbia, MD, USA; assay cut-off $=4$ dilution $^{-1}$ ).

\section{Safety}

Parents/guardians used diary cards to record solicited local and general symptoms and unsolicited adverse events. Solicited local symptoms around the injection site (pain, redness and swelling) were recorded for four days after each dose and solicited general symptoms (fever [axillary temperature $\geq 37.5{ }^{\circ} \mathrm{C} /$ rectal temperature $\geq 38^{\circ} \mathrm{C}$ ] and rash/exanthema) were recorded for 43 days after each dose. Body temperature was measured daily via the rectal/ axillary route for eight days after each vaccine dose. Between days 8-42, the presence of fever was monitored using a temperature-sensitive forehead thermometer (FeverScan ${ }^{\text {rm }}$, LCR Hallcrest, UK). If the forehead thermometer indicated fever, temperature was accurately measured using a thermometer and recorded.

The intensity of solicited symptoms was graded on a scale of $0-3$. Grade 3 solicited symptoms were defined as: child cried when limb was moved or a spontaneously painful limb (pain); injection site surface diameter $>20 \mathrm{~mm}$ (redness and swelling); axillary temperature $>39{ }^{\circ} \mathrm{C} /$ rectal temperature $>39.5{ }^{\circ} \mathrm{C}$ (fever) and $\geq 150$ lesions (rash/exanthema).

Unsolicited symptoms were recorded for 43 days after each dose and were classified as Grade 3 if they prevented everyday activity. Serious adverse events (SAEs) were recorded throughout the study.

\section{Statistical analysis}

All statistical analyses were performed using SAS 9.1 and Proc StatXact 7.0 [30, 31].

The primary objective of the study was to demonstrate non-inferiority of the first dose of the varicella vaccine without HSA when compared to the licensed vaccine. This was achieved if the lower limits (LL) of the two-sided standardized asymptotic $95 \%$ confidence intervals (CI) for the Geometric Mean Titre (GMT) ratio between the vaccine without HSA and the licensed vaccine was $\geq 0.5$ (measured by IFA) and the Geometric Mean Concentration (GMC) ratio between two groups was $\geq 0.67$ (measured by ELISA). The sample size calculated based on non-inferiority of immunogenicity and a sample size of approximately 100 subjects for each treatment group rendered $92 \%$ power to the analyses. The analysis of safety data was descriptive.

A central randomization system, using a minimization algorithm, provided each child with a unique treatment number. After randomization, a central blocking scheme was used to ensure that the balance between treaments was maintained.

Immunogenicity analysis was conducted on the according-to-protocol (ATP) cohort for immunogenicity, which included all subjects for whom both pre- and post-vaccination data were available for both vaccine doses, who were seronegative (anti-VZV antibody titre below the assay cut-off) for anti-VZV antibodies before vaccination, and complied with study procedures. The analysis of safety was conducted on the total vaccinated cohort (TVC) which included all subjects who received at least one dose of study vaccine.

Seroconversion (IFA data) was defined as an anti-VZV antibody titre $\geq$ the assay cut-off value $\left(4\right.$ dilution $^{-1}$ ) in previously seronegative subjects. Seroresponse (ELISA data) was defined as an antibody concentration $\geq$ the assay cutoff $(25 \mathrm{mIU} / \mathrm{mL})$ in previously seronegative subjects.

The percentage of subjects who showed seroconversion or seroresponse after both vaccine doses was calculated with $95 \%$ CI. The corresponding anti-VZV GMT/ GMC values were calculated with $95 \% \mathrm{CI}$ by taking the anti-log of the mean of the $\log$ of titre/concentration transformations. Values below the cut-off were given arbitrary values of half the cut-off for the purpose of this study. The GMT/GMC ratio was calculated using an analysis of variance. The ratio and $95 \% \mathrm{CI}$ were calculated as an exponential transformation of the values. A two-sided standardized asymptotic $95 \%$ CI was calculated on the difference in the percentage of subjects with anti-VZV antibody concentrations $\geq 50 \mathrm{mIU} / \mathrm{ml}$.

\section{Results}

\section{Baseline characteristics}

A total of 244 subjects were enrolled (122 in each group) of whom 233 were included in the ATP immunogenicity cohort (Group $A=117$; Group $B=116$; Fig. 1). The baseline characteristics were similar between the groups (Table 1). The mean age of the children in the TVC was 15.2 months (standard deviation [SD]: \pm 3.2 ), $51.2 \%$ were male, and all were Caucasian.

\section{Immunogenicity}

The proportion of initially seropositive individuals was 0.8 \% in Group A and 3.3 \% in Group B when antibody 


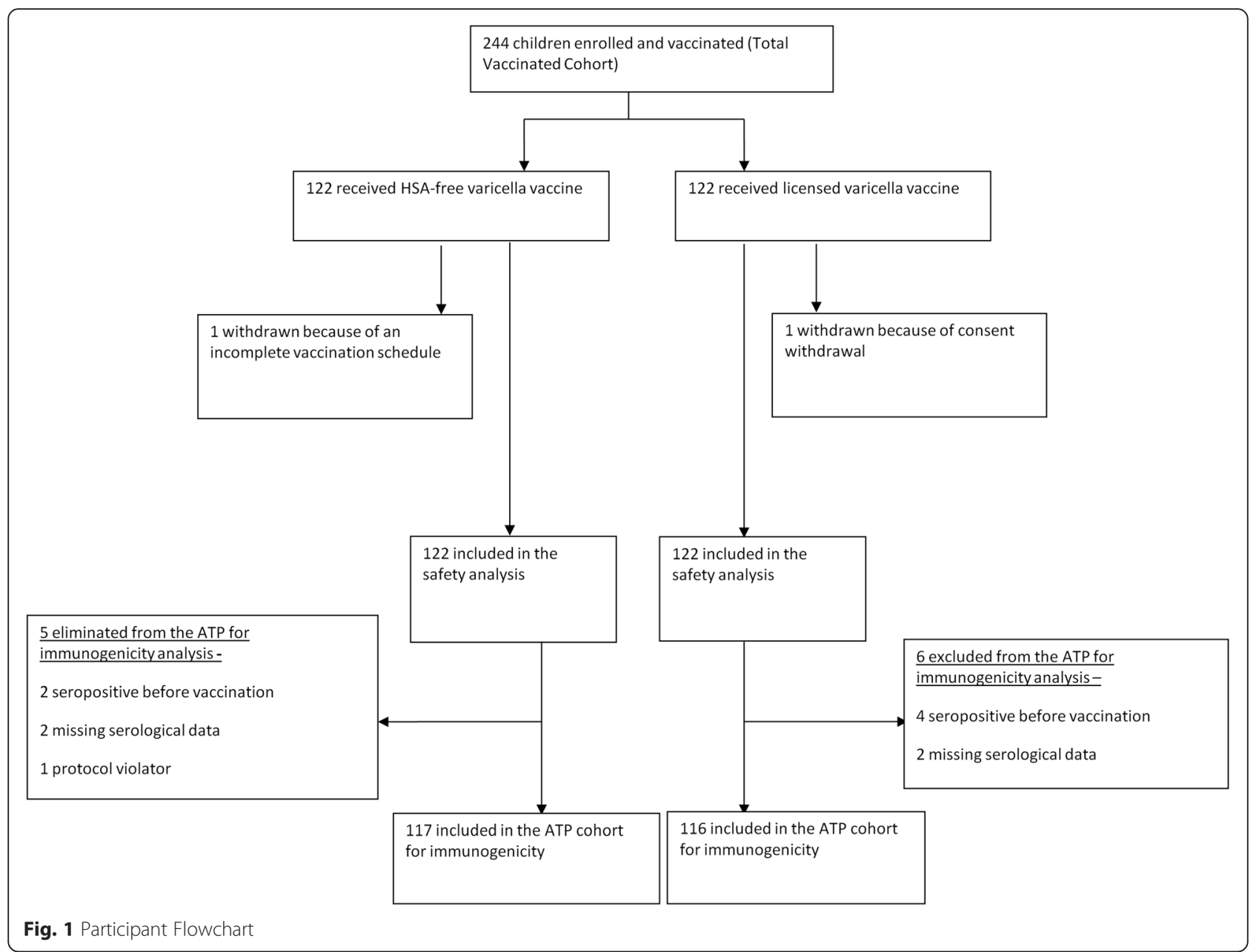

titres/concentrations were analyzed by IFA, compared with $0.8 \%$ in both groups when analysed using ELISA.

Six weeks post-dose-1, the seroconversion rates, as measured by IFA, were similar in Group A (98.3\% [95 \% CI: 93.9-99.8\%]) and B (99.1 \% [95 \% CI: 95.3$100 \%$ ]) (Table 2). Post-dose-2 seroconversion rates were $100 \%$ in both groups. After dose-1, $90.5 \%$ (95 \% CI: 83.7-95.2 \%) subjects in Group A and 89.6 \% (95 \% CI:

Table 1 Baseline characteristics (total vaccinated cohort $N=244$ )

\begin{tabular}{lllll}
\hline Characteristic & & $\begin{array}{l}\text { Group A } \\
(N=122)\end{array}$ & $\begin{array}{l}\text { Group B } \\
(N=122)\end{array}$ & $\begin{array}{l}\text { Total } \\
(N=244)\end{array}$ \\
\hline Age (months) & Mean & 15.6 & 14.8 & 15.2 \\
& SD & 3.3 & 3.1 & 3.2 \\
Gender (n [\%]) & Male & $63(51.6)$ & $62(50.8)$ & $125(51.2)$ \\
& Female & $59(48.4)$ & $60(49.2)$ & $119(48.8)$
\end{tabular}

Group A: varicella vaccine without HSA administered according to a two-dose regimen

Group B: licensed varicella vaccine administered according to a two-dose regimen

$\mathrm{N}$ : total number of subjects

n (\%): number (percentage) of subjects in each category

SD: standard deviation
82.5-94.5 \%) in Group B had concentrations of antiVZV antibodies $\geq 50 \mathrm{mIU} / \mathrm{ml}$.

Six weeks post-dose-1, the seroresponse rates as measured by ELISA, were $98.3 \%$ in Groups A and B (Table 2). Six weeks post-dose-2, the seroresponse rates were $100 \%$ in both groups.

The primary non-inferiority criteria post-dose- 1 was achieved since the LL of the $95 \%$ CI between the vaccine without HSA and licensed varicella vaccine were: 0.86 for the GMT ratio by IFA (i.e. $\geq$ the pre-defined clinical limit of 0.5 ); and 0.93 for the GMC ratio by ELISA (i.e. $\geq$ the pre-defined clinical limit of 0.67).

\section{Safety and reactogenicity}

The incidence of solicited and unsolicited symptoms, 43 days post-dose- 1 was: $76 \%$ in Group A and $69.7 \%$ in Group B; post-dose-2, the incidence was 71.9 and $68.6 \%$, respectively.

Injection site redness was the most common solicited local symptom (any and Grade 3) in both groups after both doses (Fig. 2). During the eight days of active follow-up after dose-1 when temperature was monitored 
Table 2 Immunogenicity of vaccine without HSA and licensed varicella vaccines six weeks after doses 1 and 2 (according-toprotocol immunogenicity cohort $N=233$ )

\begin{tabular}{|c|c|c|c|c|c|c|c|}
\hline \multirow[t]{2}{*}{ Dose } & \multicolumn{3}{|c|}{$\begin{array}{l}\text { Group A } \\
\text { Varicella vaccine without HSA }\end{array}$} & \multicolumn{3}{|c|}{$\begin{array}{l}\text { Group B } \\
\text { Licensed varicella vaccine }\end{array}$} & \multirow{2}{*}{$\begin{array}{l}\text { Ratio (Group A/Group B) } \\
\text { Post-dose } 1 \\
\text { GMT (95 \% Cl) }\end{array}$} \\
\hline & $\bar{N}$ & SC \% (95 \% Cl) & GMT $(95 \%$ Cl) & $\bar{N}$ & SC (95\% Cl) & GMT (95 \% Cl) & \\
\hline \multicolumn{8}{|l|}{ IFA } \\
\hline 6 weeks Post-dose 1 & 116 & $98.3(93.9 ; 99.8)$ & $172.6(141.6 ; 210.3)$ & 115 & $99.1(95.3 ; 100)$ & 154.3(128.7; 185.0) & \multirow[t]{2}{*}{$1.12(0.86 ; 1.46)$} \\
\hline 6 weeks Post-dose 2 & 115 & $100(96.8 ; 100)$ & $1452.5(1240.7 ; 1700.5)$ & 112 & $100(96.8 ; 100)$ & $1395.4(1183.0 ; 1645.9)$ & \\
\hline Dose & $N$ & SR (95 \% Cl) & GMC (95 \% Cl) & N & SR $(95 \%$ Cl) & GMC (95 \% Cl) & GMC (95 \% Cl) \\
\hline \multicolumn{8}{|l|}{ ELISA } \\
\hline 6 weeks Post-dose 1 & 116 & $98.3(93.9 ; 99.8)$ & 123.5(107.9; 141.4) & 115 & $98.3(93.9 ; 99.8)$ & $110.7(98.4 ; 124.6)$ & \multirow[t]{2}{*}{$1.12(0.93 ; 1.33)$} \\
\hline 6 weeks Post-dose 2 & 116 & $100(96.9 ; 100)$ & $1013.6(880.9 ; 1166.4)$ & 114 & $100(96.8 ; 100)$ & $999.2(877.3 ; 1138.1)$ & \\
\hline
\end{tabular}

$\mathrm{N}$ : total number of subjects

$95 \%$ Cl: $95 \%$ confidence interval

SC \%: seroconversion rate

SR \%: Seroresponse rate

GMT: geometric mean antibody titre calculated on all subjects by IFA

GMC: geometric mean antibody concentration calculated on all subjects by ELISA

Criteria for non-inferiority:

The lower limit of the $95 \%$ confidence interval $(\mathrm{Cl})$ for the GMT ratio (derived by IFA) between Group A and Group B is equal to or above the pre-defined clinical limit of 0.5

The lower limit of the $95 \% \mathrm{Cl}$ for the GMC ratio (derived from ELISA) between Group A and Group B is equal to or above the pre-defined clinical limit of 0.67

daily, the fever rate in Group A was 28.1 \% (95 \% CI: 20.3$37.0 \%$ ) and $18.0 \%$ (95 \% CI: 11.7-26.0 \%) in Group B. Post-dose-1, Grade 3 fever occurred in four subjects in Group A and two subjects in Group B. Post-dose-2, fever was recorded in $25.6 \%$ (95 \% CI: 18.1-34.4\%) of Group A subjects and $19 \%$ (95 \% CI: 12.4-27.1\%) of Group B subjects. Grade 3 fever, post-dose-2 occurred in one subject in Group A and two subjects in Group B. The observed incidence of fever during the 43 days of follow up postdose- 1 was also numerically higher in Group A (52.9 \% [95 \% CI: 43.6-62 \%]) as compared with Group B (42.6\% [95 \% CI: 33.7-51.9 \%]) (Fig. 2), although the exact $95 \%$ CIs rates were overlapping.

During the 43-day follow-up period post-dose-1, rash/exanthema was seen in $2.5 \%$ (95\% CI: $0.5-7.1 \%)$ subjects in Group A subjects compared with $3.3 \%$

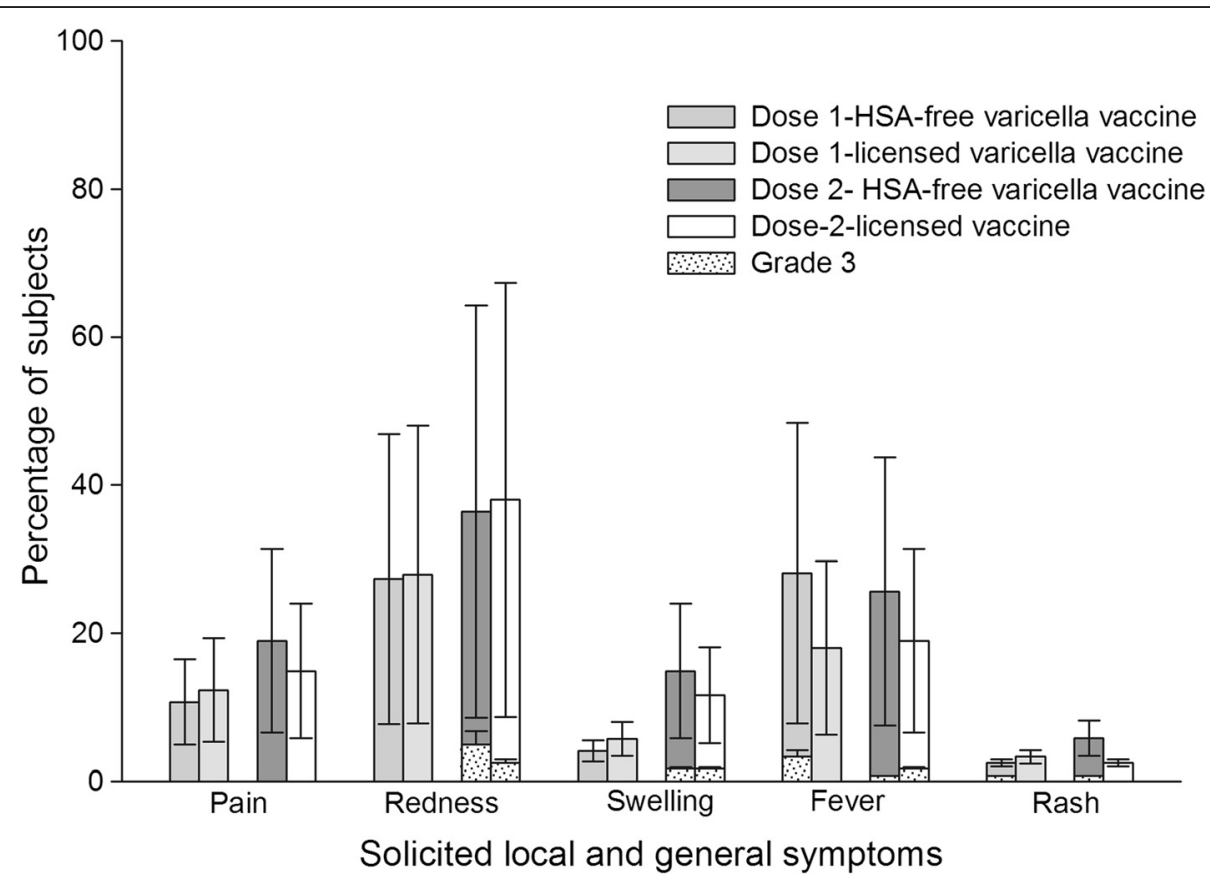

Fig. 2 Solicited local (4-days post-vaccination) and general symptoms (43 days post-vaccination) (Total Vaccinated Cohort $N=244$ ). \%: percentage of subjects reporting the symptom at least once. $95 \%$ Cl: Exact $95 \%$ confidence interval; Grade 3 pain: Cried when limb was moved/spontaneously painful; Grade 3 Redness/swelling $>20.0$ mm; Grade 3 Fever $>39.0^{\circ} \mathrm{C}$; Grade 3 Rash $>150$ lesions 
(95 \% CI: $0.9-8.2 \%$ ) in Group B. During the 43-day follow-up period after the second dose, the observed incidence of any type of rash was $5.8 \%$ (95 \% CI: 2.411.6 \%) in Group A and $2.5 \%$ (95 \% CI: $0.5-7.1 \%)$ in Group B (Fig. 2).

At least one unsolicited symptom was reported in 45.9 \% Group A versus 36.9 \% Group B subjects during the 43-days post-dose-1 follow-up period. The most common unsolicited symptom in Group A was bronchitis $(12.3 \% ; n=15)$ and viral infection with fever in Group B $(9.0 \% ; n=11)$. Over the 43-day post-dose 2 follow-up period, unsolicited symptoms were recorded in 28.9 and $34.7 \%$ of subjects in Groups A and B, respectively. The most common symptom was bronchitis (6.6 \% [ $n=8]$ and $9.1 \%[n=13]$, respectively).

Seven subjects, two in Group A and five in Group B recorded the following SAEs: otitis media and laryngitis (each accounting for two cases), gastroenteritis, concussion, bronchitis, breath holding attack, mastoiditis and sinusitis. One child experienced three SAEs (mastoiditis, otitis media and sinusitis). None of the SAEs were fatal and all were considered unrelated to vaccination.

\section{Discussion}

The European Medicines Agency has advised vaccine manufacturers to gradually eliminate the use of blood derived products of human origin because of a theoretical risk of contamination $[27,32]$. This phase II study conducted in the Czech Republic and Hungary compared the immunogenicity and safety of a varicella vaccine without HSA (developed to comply with the EMA guidance) with that of the licensed varicella vaccine in children below two years of age. After one dose, the immune responses elicited by the varicella vaccine without HSA were non-inferior to those elicited by the licensed varicella vaccine, i.e. the LL of the two-sided standardized asymptotic $95 \% \mathrm{CI}$ for the between-vaccine IFA-measured GMT ratio was $\geq 0.5$ and for the ELISA-measured GMC ratio was $\geq 0.67$.

One of the most important characteristics for paediatric vaccine development is safety, especially as it is essential to maintain high vaccination coverage to control disease.

We utilized two assays in this study: IFA, the serologic method used to license the original varicella vaccine and ELISA. The latter test has improved performance characteristics over IFA, i.e. ELISA is more sensitive and can detect lower titres in samples [33, 34]; data from this study could also be used to support a switch to ELISA $[33,34]$. In the current study, 43 days after both doses of each vaccine were administered, the seroconversion/seroresponse rates for anti-VZV antibodies were above $98 \%$, irrespective of which assay was used. These rates are consistent with pre-licensure European studies with the licensed varicella vaccine in which age-stratified children (15 months-2 years and 2-6 years) achieved average seroconversion rates of $97 \%$ [35] and a more recent study from Indonesia where children aged 10 months- 12 years demonstrated a seroconversion rate of $97 \%$ to the licensed varicella vaccine [36].

A numerical (but not statistically significant) increase in the incidence of fever was observed following the administration of the first dose of the vaccine without HSA as compared to the HSA-containing vaccine. This difference appeared to be driven by an increase in fever in the group receiving the vaccine without HSA as compared to the HSA-containing group within eight days post-vaccination. The difference was only observed in low grade fever; no difference in high grade fever $\left(>39^{\circ} \mathrm{C}\right)$ was noted. It is unlikely that this increase in low grade fever might have any significant clinical implications.

The results of this study should be interpreted with caution. First, the comparison between IFA and ELISA assays was not an objective of this study and therefore is primarily descriptive. Second, the sample size was not calculated for the analysis of safety and while we discuss the differences in fever rates between the two vaccines, we cannot draw strong conclusions based on it. Third, administering varicella vaccines with other routine vaccines would have presented a more complete view and further research may be required.

\section{Conclusion}

The first dose of a new varicella vaccine without HSA was immunologically non-inferior to the licensed varicella vaccine. Two doses of the varicella vaccine had an acceptable safety profile in children aged 11-21 months in the Czech Republic and Hungary. We utilized two assays in this study because the ELISA test has reputed improved performance characteristics over the IFA and to support a future switch to ELISA.

\section{Abbreviations \\ ATP: According-to-protocol; Cl: Confidence interval; ELISA: Enzyme linked immunosorbent assay; GMC: Geometric mean concentration; \\ GMT: Geometric mean titre; HSA: Human serum albumin; HZ: Herpes zoster; IFA: Immunoflourescence assay; LL: Lower limit; SAE: Serious adverse events; TVC: Total vaccinated cohort; VZV: Varicella-zoster virus.}

\section{Competing interests}

Dr. Roman Prymula received grants for conducting this trial and personal fees from the GSK group of companies, and also received grants from Pfizer, Sanofi-Pasteur, Baxter, Merck \& Co., Inc and GSK group of companies outside the scope of the submitted work. Dr. Andrea Kulcsar received payment for lectures and articles from Novartis, Pfizer, Sanofi-Pasteur and the GSK group of companies. Michael Povey is an employee of the GSK group of companies. Dr. Robert Simko received payment as the principal investigator of the clinical trial.

\section{Authors' contribution}

$\mathrm{RP}, \mathrm{AK}$ and RS were investigators on the clinical trial. RP and AK conceptualized and designed the study as well as assembling and collecting data. RP was also involved in choosing the centres and recruiting investigators. RS participated in interpreting the data. MP supervised the analysis and provided statistical expertise to interpret the data. All authors critically reviewed and approved the manuscript at all stages of development. 


\section{Acknowledgments}

The authors would like to thank all the investigators involved in this trial. In addition, the authors acknowledge Ouzama Henry and Carmen Baccarini for their expert input and critical review of the manuscript, Preethi Govindarajan for medical writing, Julia Donnelly (freelance on behalf of GSK group of companies) for language editing, and Shruti MP and Ashmita Ravishankar for publication coordination (employed by GSK group of companies).

\section{Funding}

This study was sponsored and funded by GlaxoSmithKline Biologicals SA Rixensart, Belgium. GlaxoSmithKline Biologicals SA was involved in all stages of the study conduct and analysis and also took charge of all costs associated with the development and the publishing of this manuscript.

\section{Trademark}

Virgo is a registered trademark of Hemagen Diagnostics, Columbia, MD, USA. Enzygnost is a registered trademark of Dade Behring, Marburg, Germany Varilrix is a registered trademark of GSK group of companies Varivax is a registered trademark of Merck \& Co.In, USA

\section{Author details}

'Faculty of Military Health Sciences, University of Defence, Trebesska 1575, 50001 Hradec Kralove, Czech Republic. ${ }^{2}$ Primary Care Paediatric Praxis, No 8, Miskolc, Hungary. ${ }^{3}$ GSK Vaccines, Avenue Fleming 20 B-1300, Wavre, Belgium. ${ }^{4}$ Szent László Hospital, Gyali Street 5-7, Budapest 1097, Hungary.

Received: 28 November 2014 Accepted: 8 January 2016

\section{Published online: 13 January 2016}

\section{References}

1. Lopez A, Schmid S, Bialek S: VPD Surveillance Manual: Varicella [http://www. cdc.gov/vaccines/pubs/surv-manual/chpt17-varicella.pdf]. Accessed 21 May, 2014.

2. Vaccines and Immunizations: Varicella [http://www.cdc.gov/vaccines/pubs/ pinkbook/varicella.html]. Accessed 21 May, 2014.

3. Shapiro M, Kvern B, Watson P, Guenther L, McElhaney J, McGeer A. Update on herpes zoster vaccination: a family practitioner's guide. Can Fam Physician. 2011:57:1127-31

4. Marin M, Güris D, Chaves SS, Schmid S, Seward JF, Advisory Committee on Immunization Practices, Centers for Disease Control and Prevention (CDC). Prevention of varicella: recommendations of advisory committee on immunization practices (ACIP). MMRV Recomm Rep. 2007:56:1-40.

5. Aebi C, Fischer K, Gorgievski M, Matter L, Muhlemann K. Age-specific seroprevalence to varicella-zoster virus: study in Swiss children and analysis of European data. Vaccine. 2001;19:3097-103.

6. Gabutti G, Penna C, Rossi M, Salmaso S, Rota MC, Bella A, et al. The seroepidemiology of varicella in Italy. Epidemiol Infect. 2001;126:433-40.

7. Kanra G, Tezcan S, Badur S, Turkish National Study Team. Varicella seroprevalence in a random sample of the Turkish population. Vaccine. 2002:20:1425-8

8. Schmitt HJ, Booy R, Weil-Olivier C, Van Damme P, Cohen R, Peltola H. Child vaccination policies in Europe: a report from the summits of independent european vaccination experts. Lancet Infect Dis. 2003;3:103-8.

9. Thiry N, Beutels P, Shkedy Z, Vranckx R, Vandermeulen C, Wielen MV, et al. The seroepidemiology of primary varicella-zoster virus infection in Flanders (Belgium). Eur J Pediatr. 2002;161:588-93.

10. Sengupta N, Booy R, Schmitt HJ, Peltola H, Van-Damme P, Schumacher RF, et al. Varicella vaccination in Europe: are we ready for a universal childhood program? Eur J Pediatr. 2008:167:47-55.

11. Ciofi Degli Atti ML, Salmaso S, Bella A, Arigliani R, Gangemi M, Chiamenti G, et al. Pediatric sentinel surveillance of vaccine-preventable diseases in Italy. Pediatr Infect Dis J. 2002;21:763-8.

12. Pinot De Moira A, Nardone A, ESEN2 Group. Varicella Zoster Virus Vaccination Policies and Surveillance Strategies in Europe. Euro Surveill. 2005;10:43-4.

13. Gershon A, Takahashi M, Seward J. Varicella vaccine. In: Plotkin SA, Orenstein WA, editors. Vaccines. 4th ed. Philadelphia (PA): Saunders; 2004. p. 783-825.

14. Skull SA, Wang EE. Varicella vaccination: a critical review of the evidence. Arch Dis Child. 2001;85:83-90.

15. Prymula R, Bergsaker MR, Esposito S, Gothefors L, Man S, Snegova N, et al. Protection against varicella with two doses of combined measles-mumps- rubella-varicella vaccine versus one dose of monovalent varicella vaccine: a multicentre, observer-blind, randomised, controlled trial. Lancet. 2014;383: 1313-24.

16. World Health Organization. The WHO position paper on varicella vaccines. Wkly Epidemiol Rec. 1998;73:241-8.

17. Macartney KK, Burgess MA. Varicella vaccination in Australia and New Zealand. J Infect Dis. 2008;197 Suppl 2:S191-5.

18. National Advisory Committee on Immunization. National Advisory Committee on Immunization (NACl) update on varicella. Can Commun Dis Rep. 2004;30:1-26

19. Childhood immunization calendar, Greece [http://vaccine-schedule.ecdc europa.eu/Pages/Scheduler.aspx]. Accessed 9 May, 2014.

20. Sadzot-Delvaux C, Rentier B, Wutzler P, Asano Y, Suga S, Yoshikawa T, et al. Varicella vaccination in Japan, South Korea and Europe. J Infect Dis. 2008; 197:185-90.

21. Quian J, Ruttimann R, Romero C, Dall'orso P, Cerisola A, Breuer T, et al. Impact of universal varicella vaccination of one year-olds in Uruguay: 1997-2005. Arch Dis Child. 2008:93:845-50.

22. WHO vaccine preventable disease monitoring system: Immunization schedules by antigen, selection centre [http://apps.who.int/immunization_ monitoring/globalsummary/schedules]. Accessed 9 May, 2014.

23. Liao SL, Huang T, Huang YC, Jiang DD. Survey of the status of self-paid varicella vaccination among children one to six years of age in Taiwan. J Microbiol Immunol Infect. 2007:40:112-5.

24. Reynolds MA, Watson BM, Plott-Adams KK, Jumaan AO, Galil K, Maupin TJ, et al. Epidemiology of varicella hospitalizations in the United States, 1995-2005. J Infect Dis. 2008;197:120-6.

25. Kreth HW, Lee BW, Kosuwon P, Salazar J, Gloriani-Barzaga N, Bock HL, et al. Sixteen years of global experience with the first refrigerator-stable varicella vaccine (Varilrix). BioDrugs. 2008:22:387-402

26. Offit PA, Jew RK. Addressing parents' concerns: do vaccines contain harmful preservatives, adjuvants, additives, or residuals? Pediatr. 2003;112:1394-7.

27. EMA: The European Agency for the Evaluation of Medicinal Products: Note for Guidance on Plasma-Derived Medicinal Products. 2001. [http://www. ema.europa.eu/docs/en GB/document library/Scientific quideline/2009/09/ WC500003613.pdf] Accessed 7 October, 2015.

28. Lau YL, Vessey SJ, Chan IS, Lee TL, Huang LM, Lee CY, et al. A comparison of safety, tolerability and immunogenicity of Oka/Merck varicella vaccine and VARILRIX ${ }^{\mathrm{TM}}$ in healthy children. Vacc. 2002;20(23-24):2942-9.

29. Schuster V, Otto W, Maurer L, Tcherepnine P, Pfletschinger U, Kindler K, et al. Immunogenicity and Safety Assessments After One and Two Doses of a Refrigerator-Stable Tetravalent Measles-Mumps-Rubella-Varicella Vaccine in Healthy Children During the Second Year of Life. Pediatr Infect Dis J. 2008; 27(8):724-30.

30. Clopper CJ, Pearson ES. The use of confidence or fiducial limits illustrated in the case of binomial. Biometrika. 1934:26:404-13.

31. Newcombe RG. Interval estimation for the difference between independent proportions: Comparison of eleven methods. Statist Med. 1998;17:873-90.

32. EMEA-CHMP. EMEA-CHMP: Position Statement on Creutzfeldt-Jakob Disease and Plasma-derived and Urine-derived Medicinal Products (2004). [http://www.ema.europa.eu/docs/en_GB/document_library/Position_ statement/2011/06/WC500108071.pdf]. Accessed 9 May, 2014.

33. Deedler AM, Kornelis D. A comparison of the IFA and the ELISA for the demonstration of antibodies against Schistosome gut-associated polysaccharide antigens in Schistosomiasis. Parasite Res. 1980:64:65-75.

34. Yahara Y, Okhubo Y, Kariwa H, Takashima I. Evaluation of enzyme-linked immunosorbent assay (ELISA) and immunofluorescent antibody (IFA) test for the detection of porcine reproductive and respiratory syndrome virus (PRRSV) antibody in pigs from conventional farms. J Vet Med Science. 2002;64:583-8.

35. Gillet $Y$, Steri GC, Behre U, Arsène JP, Lanse X, Helm K, et al. Immunogenicity and safety of measles-mumps-rubella-varicella (MMRV) vaccine followed by one dose of varicella vaccine in children aged 15 months-2 years or 2-6 years primed with measles-mumps-rubella (MMR) vaccine. Vaccine. 2009;27:446-53.

36. Hadinegoro SR, Hindra IS, Han HH, Gatchalian S, Bock HL. Reactogenicity and immunogenicity of a live-attenuated refrigerator-stable varicella vaccine (OKA strain) in healthy seronegative children age 10 months to 12 years. Southeast Asian J Trop Med Public Health. 2009:40:991. 